\title{
ARTICLE
}

\section{In-vivo single neuron axotomy triggers axon regeneration to restore synaptic density in specific cortical circuits}

\author{
A. J. Canty ${ }^{1, \dagger}$, L. Huang ${ }^{1, \dagger}$, J. S. Jackson ${ }^{1, \dagger}$, G. E. Little ${ }^{1}$, G. Knott ${ }^{2}$, B. Maco ${ }^{2} \&$ V. De Paola ${ }^{1}$
}

To what extent, how and when axons respond to injury in the highly interconnected grey matter is poorly understood. Here we use two-photon imaging and focused ion beamscanning electron microscopy to explore, at synaptic resolution, the regrowth capacity of several neuronal populations in the intact brain. Time-lapse analysis of $>100$ individually ablated axons for periods of up to a year reveals a surprising inability to regenerate even in a glial scar-free environment. However, depending on cell type some axons spontaneously extend for distances unseen in the unlesioned adult cortex and at maximum speeds comparable to peripheral nerve regeneration. Regrowth follows a distinct pattern from developmental axon growth. Remarkably, although never reconnecting to the original targets, axons consistently form new boutons at comparable prelesion synaptic densities, implying the existence of intrinsic homeostatic programmes, which regulate synaptic numbers on regenerating axons. Our results may help guide future clinical investigations to promote functional axon regeneration.

\footnotetext{
${ }^{1}$ MRC Clinical Science Centre, Faculty of Medicine, Imperial College London, London W12 ONN, UK. ${ }^{2}$ Centre of Interdisciplinary Electron Microscopy, École Polytechnique Fédérale de Lausanne, CH1015 Lausanne, Switzerland. †Present addresses: Wicking Dementia Research and Education Centre, University of Tasmania, Hobart, Australia (A.J.C.); Australian Regenerative Medicine Institute, Monash University, Clayton VIC 3800, Australia (L.H.); Lilly UK, Windlesham, Surrey, UK (J.S.J.). Correspondence and requests for materials should be addressed to V.D.P. (email: vincenzo.depaola@imperial.ac.uk).
} 
P lasticity of the adult injured central nervous system (CNS) may involve structural changes, such as sprouting of de novo axonal branches, regeneration at the severed axonal stump or synaptic remodelling. Major efforts in this field have so far concentrated on the spinal cord and white matter injury models ${ }^{1-3}$, with much less known about these repair mechanisms in the grey matter of the brain. Unlike the peripheral nervous system, it is believed that the adult CNS does not mount a significant spontaneous regenerative attempt to reconnect with prelesion targets due to both intrinsic (to the neuron) and extrinsic inhibitory (glial-derived) factors ${ }^{1}$. However, the relative contribution of these mechanisms has been difficult to disentangle in the intact brain ${ }^{4}$, leaving open basic questions regarding the endogenous capacity for repair. For example, can axons regenerate in the absence of glial scar-mediated inhibition, reach the prelesion targets and form synapses to recover the lost output? Does the pattern of any regrowth recapitulate developmental axon growth or does it employ a distinct growth programme? These questions are particularly important now that many experimental manipulations can successfully initiate axon regeneration in the adult injured $\mathrm{CNS}^{5-7}$. We hypothesized that axons that normally display a high rate of synaptic and branch remodelling, that is, from Layer (L) 6 cortical cells would more readily respond to a lesion than axons that are typically more stable, that is, thalamocortical axons (TCA) projecting to the upper layers of the cortex ${ }^{8}$. This intrinsically specified plasticity is reminiscent of the differences in regeneration between different axonal tracts following injury ${ }^{9-11}$.

A direct comparison of the regrowth potential of different neuronal populations in the adult CNS, however, is lacking. One of the main reasons is that it is often not easy to distinguish between growth of damaged neurons (that is, regeneration) from growth of nearby spared neurons ${ }^{1,12}$. The variability of results due to the difficulty of controlling both the extent of any lesion and inflammation is also a major confounding factor in assessing reorganization and repair therapies. Most previous studies have investigated these properties in fixed preparations, which make the distinction between regeneration and growth from spared fibres difficult, and can provide only minimal insights into key dynamic features. Similarly, studies conducted in vitro, which are useful to dissect mechanisms of axon degeneration and regeneration, may artificially influence any amount of regrowth observed. In particular, no previous study could investigate the complex asynchronous and dynamic features of individual lesioned axon responses in the intact mammalian brain.

To overcome these limitations we have used a combination of two-photon (2P) microscopy, laser microsurgery and retrospective focused ion beam-electron microscopy (EM) to monitor the response of excitatory cortical circuitry for periods ranging from minutes up to a year in the brain of living mice and in the absence of glial scars. Our data unequivocally provide in vivo evidence for spontaneous circuit-specific axon regeneration and for the existence of neuron-intrinsic homeostatic programmes, which regulate synaptic numbers on regenerating axons. They further suggest that for those axon subclasses, which are unable to regrow, this is due to a neuron-intrinsic inability rather than gliamediated inhibition.

\section{Results}

Laser-mediated axotomy in the living mouse brain. To assess the relative contribution of extrinsic versus intrinsic factors in regulating axon regeneration in the upper layers of the cerebral cortex we used Thy1-GFP mice, which express green fluorescent protein (GFP) in either the cytosol ${ }^{13}$ or cell membrane ${ }^{14}$ of a subset of excitatory axons with cell bodies located in L2/3/5/6 and the underlying thalamus. Axons were imaged individually through a cranial window and classified according to their morphology based on previous anatomical reconstructions and findings ${ }^{8}$ (see Methods). To study axonal reorganization without the confounding factors of inflammation and glial-mediated inhibition we used 2P laser-mediated axotomy, which leaves the dura mater unperturbed, and is associated with minimal scarring and immune response ${ }^{15-17}$.

Typically, long stretches of axons (up to $2 \mathrm{~mm}$ ) could be identified in the upper layers of the cortex (Fig. 1a). We were able to transect axons within defined lengths of their axon tips in most neurons (for 58 out of 83 axons, mean portion cut $=542 \pm 38 \mu \mathrm{m}$, range $\sim 150-1,289 \mu \mathrm{m})$. Care was taken to lesion away from large vessels to avoid any confounding effects of bleeding or inflammation (Fig. 1b). By manipulating the parameters of the excitation laser, we were able to reproducibly induce localized axonal lesions (Fig. 1c), which resulted in the generation of a fluorescent mark (diameter $=4.13 \pm 0.32 \mu \mathrm{m}, n=10$ lesions) in the tissue (Fig. 1c). The eventual disappearance of the disconnected distal axon segment in all cases (107 in total; 83 in the adult brain and 24 in the juvenile brain) verified that the axons were severed in each case. The axonal segment proximal to the cell body survived the lesion in all cases and was observed for up to 12 months for signs of remodelling.

Absence of glial scar after laser-mediated microlesions. We first sought to confirm that the laser microlesions were not associated with the formation of a prolonged glial reaction and persistent scar tissue (Fig. 2). We collected tissue at a series of intervals post lesion for immunohistochemical analysis of the lesion site and surrounding neuropil (Fig. 3). Expectedly, as the lesions did not target the microvasculature, we did not see a response by blood borne monocytes. The resident immune cells of the brain, the microglia, did undergo a rapid and localized yet transient response ${ }^{18}$. Using an antibody against ionized calcium binding adapter molecule 1 (Iba1), which recognizes both resting and reactive microglia (differentiated on the basis of morphology), we observed an accumulation of microglia surrounding the lesion site within $4-5 \mathrm{~h}$ post lesion (Fig. $2 \mathrm{a}, n=4$ ), which persisted at the lesion site at $24 \mathrm{~h}$ post injury (Fig. $2 \mathrm{a}, n=6$ ). This reaction had largely dissipated by 3 days (Fig. $2 \mathrm{a}, n=3$ ) and 7-8 days post lesion $(n=3)$.

To look for any astrocytic reaction we used an antibody directed towards glial fibrillary acidic protein (GFAP), an intermediate filament in the central part of the astrocytic cytoskeleton. Although there were many GFAP-positive cells in the surrounding neuropil, there was no discernible astrocytic reaction evidence by GFAP immunoreactivity-bright, shortened, thickened processes-either at the lesion site or at the damaged axon stump for up to 8 days (d) post lesion (Fig. $2 \mathrm{~b} ;+1 \mathrm{~d}, n=3$; $+2-3 \mathrm{~d}, n=2 ;+4-5 \mathrm{~d}, n=5 ;+7-8 \mathrm{~d}, n=3)$. Estimates in the literature indicate that GFAP antibodies detect only $15 \%$ of cortical astrocytes with GFAP rarely localized to the fine terminal branches furthest from the cell body ${ }^{19}$. To gain a more detailed understanding of the laser-mediated damage, we therefore used focused ion beam-scanning EM (FIB-SEM $)^{20}$. FIB-SEM reconstruction of the lesion site $1 \mathrm{~d}$ post lesion largely confirmed our immunohistochemical findings (Fig. 2c; $n=1$ mouse). The lesion site was surrounded by an envelope of microglial folds, containing cytoplasmic inclusion bodies and lysozymes (Fig. 2c). Long astrocytic projections were evident within the nearby neuropil as were swollen mitochondria with curved cisternae. These astrocytic processes were not visible with GFAP immunohistochemistry and, therefore, were likely to be terminal projections of the astrocytic cytoskeleton. The lesion 

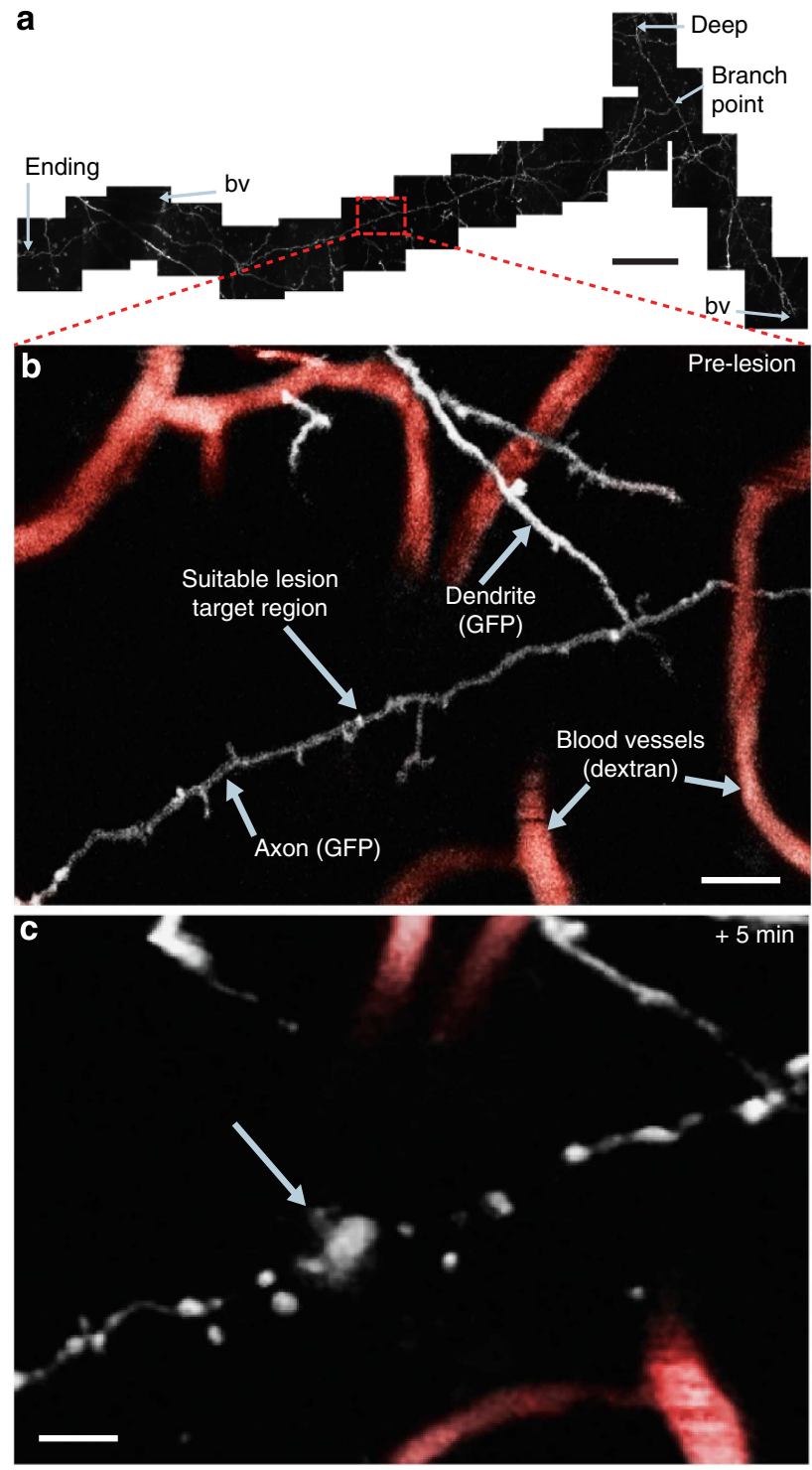

d

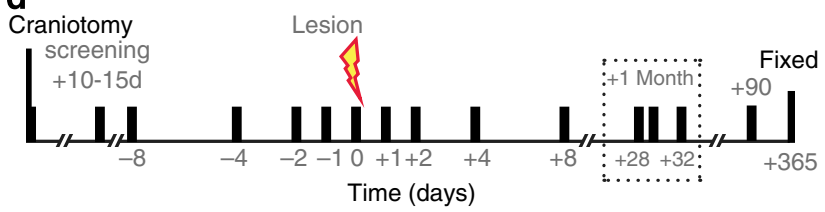

Figure 1 | Laser-mediated axotomy in the living mouse brain. (a) A typical axon in the upper layers of the cortical neuropil suitable for microlesion with this axon disappearing under a blood vessel (bv) and deep into the cortex, with a long branched axonal ending. (b) This region of the axon was identified as being ideal for microlesion and free of dextran-labelled microvasculature (indicated in red shading). (c) Same region as in a $5 \mathrm{~min}$ after a laser-mediated lesion with characteristic fluorescent mark (arrow). (d) Time course for repeated imaging pre- and post lesion. Scale bars, $100 \mu \mathrm{m}$ in $\mathbf{a}, 10 \mu \mathrm{m}$ in $\mathbf{b}$ and $5 \mu \mathrm{m}$ in $\mathbf{c}$

centre comprised of organelles, including mitochondria. Further from the lesion site the neuropil appeared normal (Supplementary Movie 1). We then assessed to what extent microglia, astrocytes and the surrounding neuropil were affected 5 days after laser damage (Fig. 2 d, $n=1$ ). EM reconstruction revealed the presence of electron dense material, which was confined to the lesion site and was surrounded by what appeared a relatively normal neuropil. We have quantified the amount of microglia and astrocytic processes around the lesion $(6 \mu \mathrm{m}$ radius) at 1 - and 5-day-old lesions. Importantly, both the microglia and astrocytic response seen after 1 day had regressed 5 days after the laser lesion (Figs 2c,d; microglia density as \% of total tissue volume: $1 \mathrm{~d}$ post lesion $=31.7 \% \pm 3.3, \quad 5 \mathrm{~d}=$ $22.4 \% \pm 1.8$; astrocyte density as $\%$ of total tissue volume: $1 \mathrm{~d}$ post lesion $=21.8 \% \pm 5.1,5 \mathrm{~d}=13.0 \% \pm 1.4 ; n=5$ serial sections for each time point), confirming our immunohistochemical findings.

We next probed for the expression of the extracellular matrix protein chondroitin sulphate (CS) proteoglycan either at the lesion site or surrounding the axonal stump. CS is a known inhibitory extracellular matrix protein secreted by astrocytes in regions of glial scarring in other injury paradigms ${ }^{21}$. We looked for CS deposition at 5d post injury, based on reverse transcription-PCR expression profiles in the literature ${ }^{22}$. There was no CS deposited at the tip of the proximal stump, nor at the lesion centre (Fig. 2e; $+5 \mathrm{~d}, n=6$ ). In summary, consistent with previous work ${ }^{15}$, laser-mediated microlesions in the cortex are not associated with a sustained prominent glial scar.

Cell type-specific axon regeneration in the adult brain. We then tested whether injured cortical axons in the grey matter are able to compensate for the reduced functional output through collateral branching or regeneration of the lesioned axonal stump in the absence of scar tissue (Fig. 4). We monitored the surviving axon shaft for up to $1 \mathrm{~mm}$ back towards the cell body and saw no sprouting of de novo collateral branches. Surprisingly, some axons observed for a minimum of 2 weeks post injury did show regrowth from the lesioned axonal stump. Regrowth was defined as any distance sufficient to reach the lesion site, provided it was at least twice the largest distance detected in the measurement noise analysis ${ }^{8}$ (see Methods). Remarkably, different neuron subclasses $^{8}$ showed variable amounts of regrowth from the severed axon ending over several weeks (mean regrowth: $125 \pm 41 \mu \mathrm{m}$, range $8-650 \mu \mathrm{m}, n=17) ; \sim 55 \%$ of $\mathrm{L} 6$ axons ( $n=12$ of 22 axons imaged for at least 2 weeks, Fig. 4a,b) attempted to regenerate compared with only $\sim 20 \%$ for all other axons, which include L2/3/5 and TCA ( $n=5$ of 26 imaged for at least 2 weeks; $P=0.01$, Pearson $\chi^{2}$-test), revealing dramatic intrinsic differences amongst neurons in the ability to regenerate. We then wondered whether the axons that did not reach the lesion site were still capable of some growth. To address this question we calculated the average maximum regrowth in a given time period as measured from the point of maximum retraction in the preceding days (Fig. 4c). Axons that never reached the lesion site $(n=29)$ grew significantly less than those axons that did reach the lesion site $(n=17)$ at 4 days post lesion $(0.48 \pm 0.25 \mu \mathrm{m}$ versus $32.98 \pm 21.54 \mu \mathrm{m}, P<0.001)$ up to 2 weeks post lesion $(1.12 \pm 0.39 \mu \mathrm{m}$ versus $15.27 \pm 5.4 \mu \mathrm{m}, P<0.001)$; and up to 6 months post lesion $(1.84 \pm 0.67 \mu \mathrm{m}$ versus $125 \pm 41.6 \mu \mathrm{m}$, $P<0.001)$. In the majority of cases, the final length of the observed regrowth was less than the amount excised by the lesion (regrowth as a fraction of the length that was removed: $33 \pm 12 \%$, range $1-180 \%, n=14$ ). Only 1 out of 48 axons monitored for periods greater than 14 days showed sufficient regeneration originating from the severed axonal stump to replace the length removed (Fig. 4b). This finding was not dependent on the amount of distal axon cut, as transecting axons within $250 \mu \mathrm{m}$ from the cell body (that is, removing $>96 \%$ of the axonal arbor) did not significantly increase the regrowth potential compared with lesions within $200-1000 \mu \mathrm{m}$ from the axon ending (that is, removing $<10 \%$ of the axonal arbor; Little and De Paola, unpublished data). These data are consistent with the idea that 

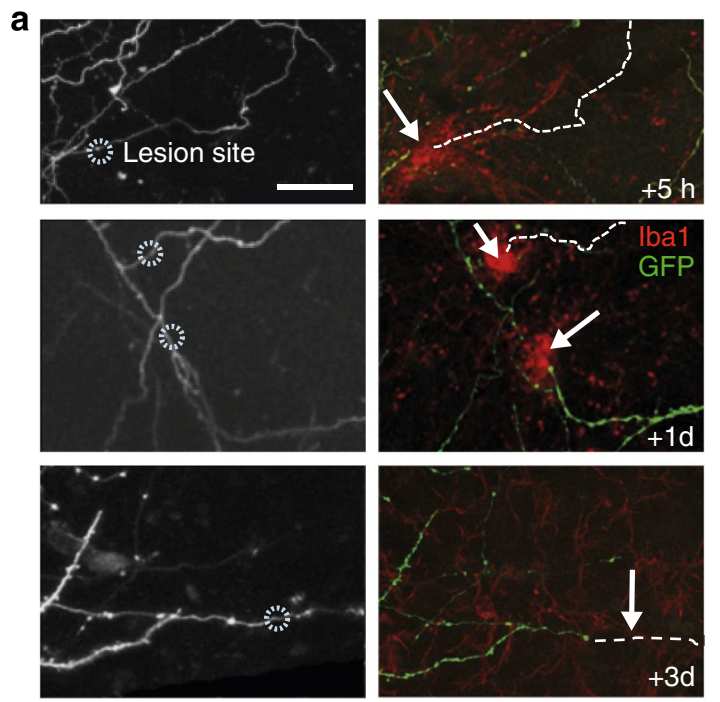

b

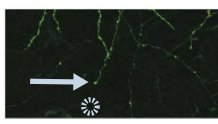

GFP
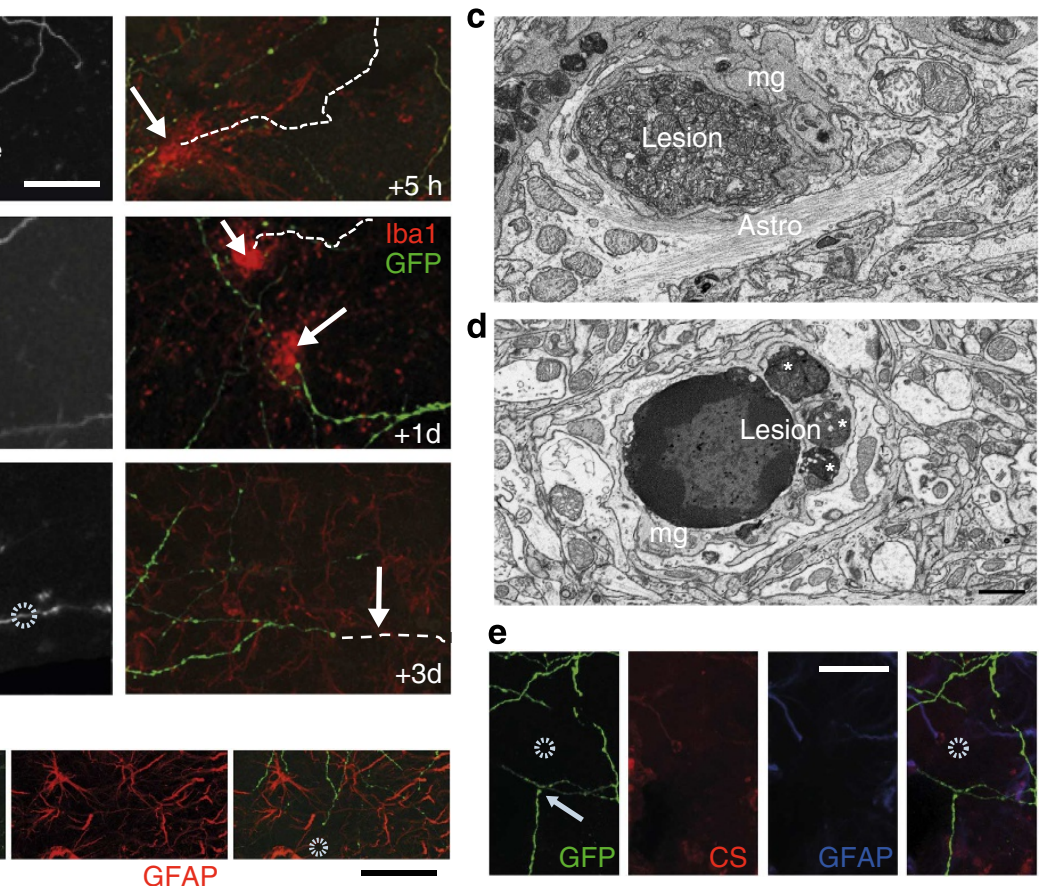

Figure 2 | Absence of a prominent glial scar following laser-mediated microlesion. (a) lba1 immunoreactive microglia (red) at $5 \mathrm{~h}$, 1d and 3d post injury. White arrows in the right panels indicate the lesion site. The dotted lines represent the trajectory of the targeted axon before the lesion (left panels). (b) GFAP (glial fibrillary acidic protein, astrocytic marker) immunohistochemistry reveals minimal association with the lesion site (circle) or axonal stump (arrow) at 3 days post lesion. The last panel shows the merged image. (c,d) FIB-SEM shows microglia (mg) and astrocytic association (Astro) with the lesion site at 1 day post lesion (c), which regresses by 5 days (d). Asterisks indicate inclusion bodies. (e) Chondroitin sulphate (CS) labelling shows no depositions at the lesion site (circle) or proximal stump (arrow) 5 days post lesion ( $n=6$ lesions). The last panel is the merge of the first three panels. Scale bars, $15 \mu \mathrm{m}$ in $\mathbf{a}, 30 \mu \mathrm{m}$ in $\mathbf{b}, 1 \mu \mathrm{m}$ in $\mathbf{c}, \mathbf{d}$ and $20 \mu \mathrm{m}$ in $\mathbf{e}$.

adult CNS neurons have a limited capacity for axonal regrowth following a lesion even in the grey matter where the inhibitory role of myelin is expected to be less prominent than in the white matter, and challenge the glial scar as the principal cause of this inability.

Regeneration is associated with prelesion bouton density. The successful regrowth of a subset of neurons enabled us to study, for the first time, the dynamic features of regrowth in the adult brain. In particular, we sought to clarify (i) the growth pattern, (ii) whether regrowing axons followed the original trajectories and (iii) whether they underwent synapse formation/elimination processes to reestablish comparable prelesion synaptic densities. This clarification is critical to assess the functional relevance of axon regrowth in the brain.

Although axonal endings in the unlesioned cortex repeatedly grow and retract by tens of microns over several days in both the maturing ${ }^{23}$ and the naive adult ${ }^{8}$ brain, regrowing injured axons typically navigated the adult environment without undergoing periods of significant retraction (Fig. 4a,b and Supplementary Movie 2) reaching a maximum extent of regrowth of $650 \mu \mathrm{m}$ over 90d post lesion (green in Fig. 4a,b and Supplementary Movie 2). This represented almost twice the length that was originally removed $(360 \mu \mathrm{m}$, red in Fig. $4 \mathrm{a})$, a distance never detected in the unlesioned adult cortex ${ }^{8}$. Occasionally, simple growth cones were observed on growing axons (Fig. 4a). The average speed of regrowing axons in the adult brain was $20 \pm 5 \mu \mathrm{m}$ per day $(n=5$ axons from 5 mice) and the fastest rate detected was $\sim 700 \mu \mathrm{m}$ per day, which was comparable to peripheral nerve regrowth ${ }^{24}$.

We hypothesized that axons in the post natal, maturing brain would also show regrowth following axotomy. To determine whether this was the case, we performed similar experiments in the juvenile cortex. We implanted cranial windows at post natal day (PND) 17-21, and then lesioned a variety of axon subtypes 7-10 days later. At this stage of development, neurons undergo dynamic growth and retraction of their axon tips, and have yet to stabilize their projections and synapses in the cortical neuropil, making it difficult to identify the origin of the axons based on morphological criteria. However, given the fact that the Thy1 promoter labels the same populations of neurons from an early postnatal age, we expect similar proportions of cell types to be labelled in our juvenile and mature adult groups ${ }^{13,8}$. In total, we injured 24 axons in 13 juvenile mice. A higher proportion of axons in the maturing brain (PND 25-31) attempted regrowth (8 out of $12(67 \%)$ compared with 17 out of $48(35 \%)$ in the adult brain). Moreover, the dynamics of regrowth in the juvenile brain were very different (Fig. 4d), with repeated cycles of growth and retraction, with regrowth essentially following the navigation behaviour of uninjured developing axons ${ }^{23}$.

Notably, in all cases of regrowth in the adult cortex, regrowth followed a different trajectory compared with the original axonal projection (Figs $4 \mathrm{a}$ and $5 \mathrm{a}-\mathrm{d}$ ).

In all cases, the regrowing axons in the adult cortex formed synaptic boutons of both types found in the cortex: terminaux boutons or en passant boutons (Figs $4 \mathrm{a}, 5$ and 6), with no regional difference in density compared with those on the prelesion axon shaft (Fig. 5e; mean prelesion density $=0.12 \pm 0.02 \mu \mathrm{m}^{-1}$; new growth density $0.17 \pm 0.03 \mu \mathrm{m}^{-1} ; n=4$ axons, $P=0.13$ ). Boutons on the regenerated axon segments make synapses (Fig. 6) and continue to be added and eliminated (Figs $4 \mathrm{a}$ and $5 \mathrm{f}$, and Supplementary Movie 2). Overall, these data open the possibility that harnessing the intrinsic growth capacity of L6 neurons could 


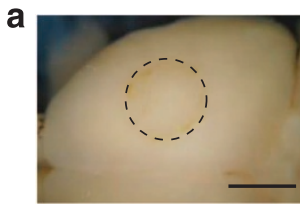

C

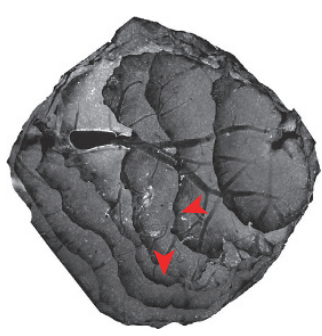

d

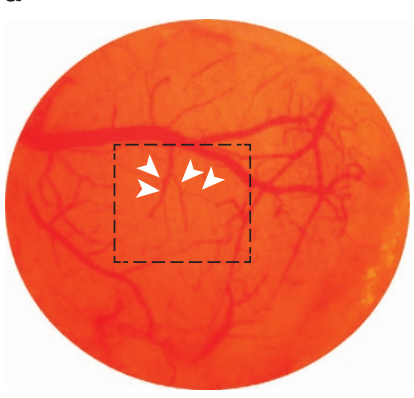

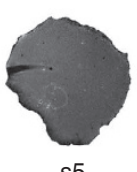

s5

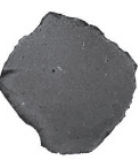

s6

e

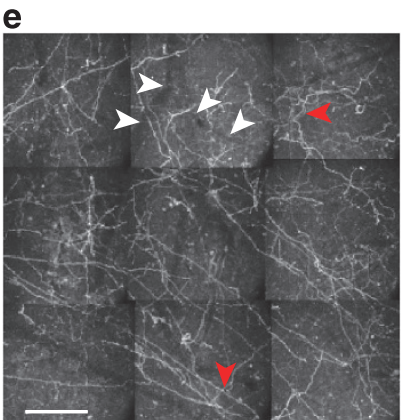

f

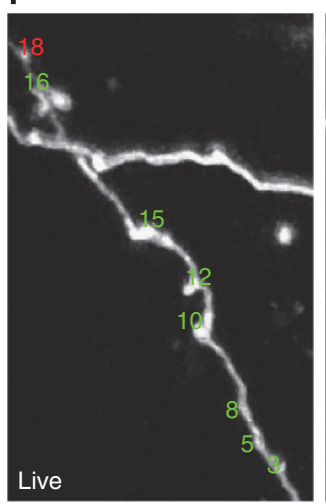

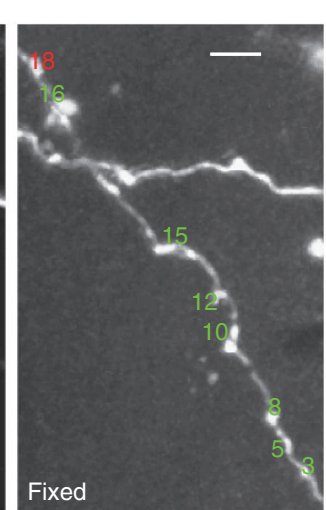

g

Figure 3 | Procedure for the identification of imaged axons in the fixed brain. (a) Low magnification of the imaged hemisphere. (b) Tangential brain sectioning. (c) Section alignment reveals the blood vessels used to align the $2 \mathrm{P}$ images. Red arrows indicate the targeted axons in e. (d) Bright-field view of superficial blood vessels from in vivo imaging. White arrows mark the same area in $\mathbf{e}$. (e) Low-magnification $2 \mathrm{P}$ overview of the boxed area in $\mathbf{d}$. Arrowheads mark the same areas in $\mathbf{c}$ and $\mathbf{d}$. (f) Terminaux Bouton (TB) annotation (green-red numbers) in live versus fixed brain. (g) There is no significant difference in TB density in live compared with fixed brain images ( $n=4$ axons, $P>0.05$, Wilcoxon's signed-rank test). Scale bar, $2.5 \mathrm{~mm}$ in $\mathbf{a}$, $50 \mu \mathrm{m}$ in $\mathbf{e}$ and $5 \mu \mathrm{m}$ in $\mathbf{f}$.

be an effective future strategy to repair injured cortical circuits in the adult brain.

\section{Discussion}

Here we have used in vivo $2 \mathrm{P}$ imaging through a cranial window on the cerebral cortex to assess the real-time reorganization of $>100$ individually injured axons at synaptic resolution and for extended periods of time in the absence of glial scars. We show that specific subclasses of axons can spontaneously regrow over distances unseen in the uninjured adult cortex and at speeds comparable to peripheral nerve regrowth. Although never retracing their original trajectories, axons consistently form synaptic boutons equalling prelesion synaptic densities, highlighting the presence of neuron-intrinsic homeostatic mechanisms, which regulate synaptic numbers. In addition, our data suggest that for those axon subclasses unable to regrow this is due to an intrinsic inability of those neurons rather than due to gliamediated inhibition.

We have used high-energy femtosecond lasers ${ }^{15-17,25,26}$ directed through the viewing window to induce localized lesions (Fig. 1). Such laser-mediated axotomy is highly reproducible and is associated with minimal scarring (Fig. 2 and refs 15,27), which means that there will be fewer confounding issues related to varying degrees of immune response or glial scar formation. Subsequently, we describe consistent effects in terms of lesion size and stereotypical axonal (for example, Fig. 1 and ref. 28) and glial (Fig. 2) responses. As we were able to follow the fate of every targeted axon over time, we could unequivocally distinguish between regrowth of damaged fibres and eventual growth of a fraction of non-damaged surrounding neurons, a limitation of other published injury paradigms. As such, we have gained unique insights into axon and synaptic remodelling following grey matter degeneration ${ }^{28}$. We expect this approach to be useful to test in vivo repair strategies in ways not previously possible in the mammalian brain.

We investigated the regenerative potential of cortical neurons in response to minimal injury, to ask how individual cortical axons respond to damage and which axonal subclasses are growth competent in vivo. To provide the first quantitative analysis of axonal reorganization after focal injury in the brain of living mice we focused on the dynamics of surviving L6, L2/3/5 and TCA segments. We have previously shown that under control conditions these cortical axons in the adult brain do not extensively remodel, displaying only minor $(\sim 5 \%)$ length changes over 1 month $^{8}$. Except for L6 axons, which can 

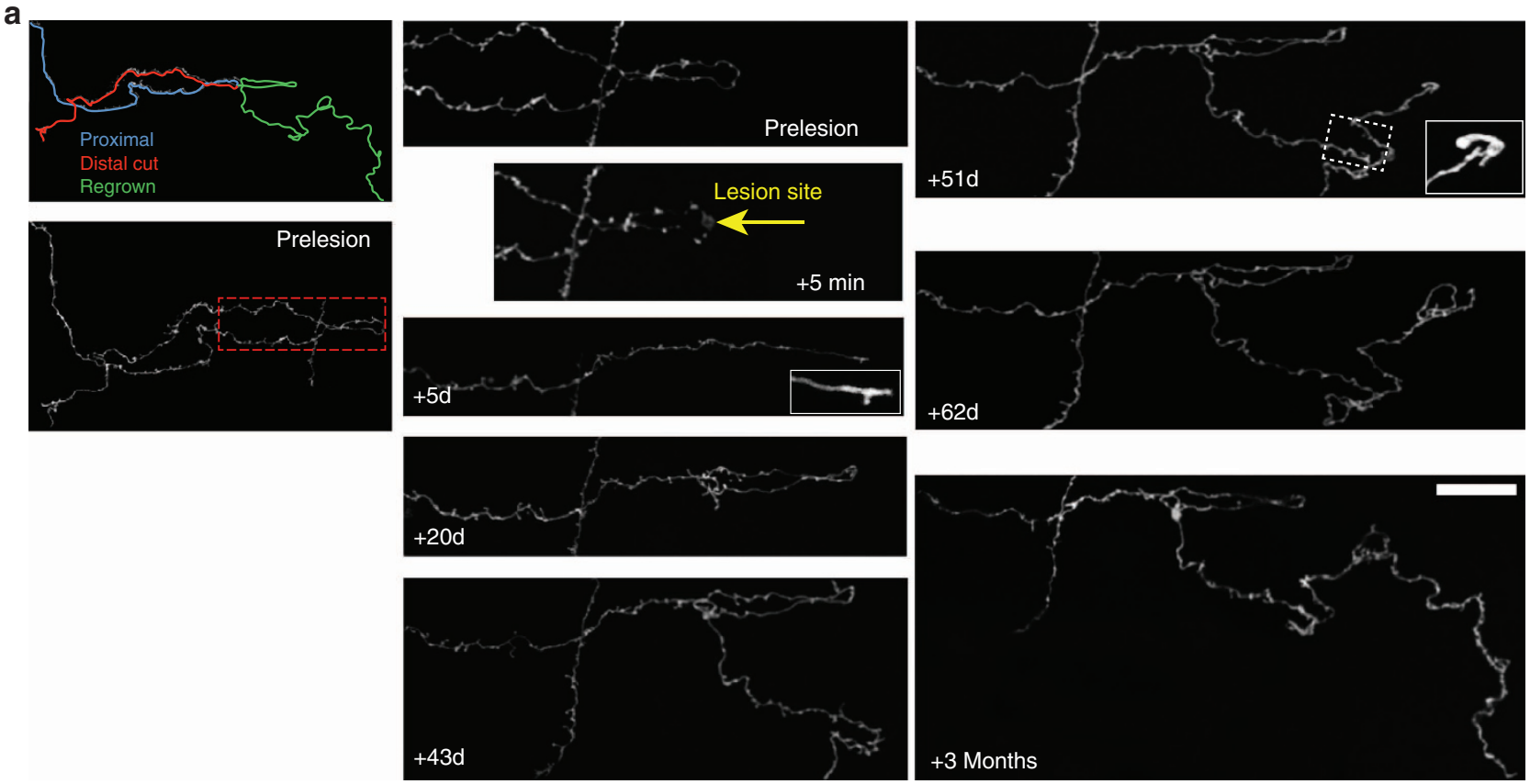

b

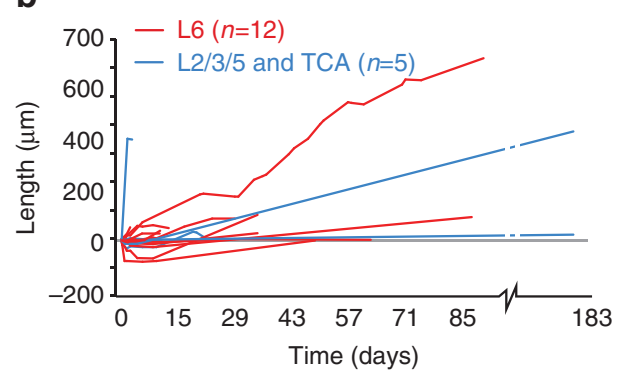

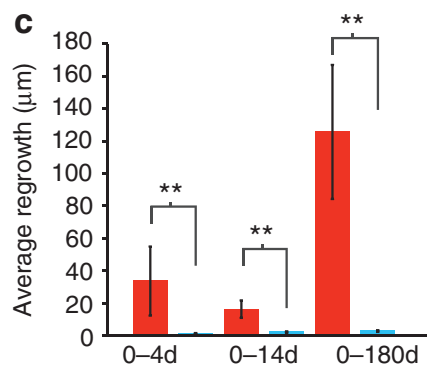

- Axons that did not reach the lesion site

axons that reached the lesion site

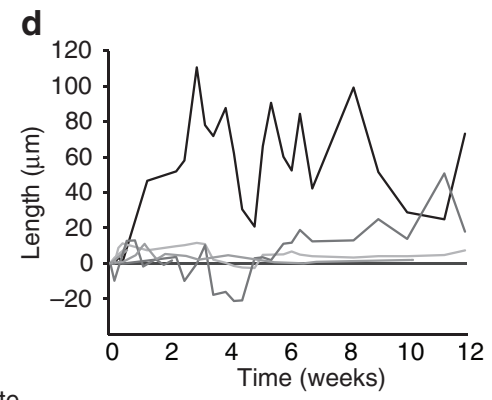

Figure 4 | Cell type-specific axon regeneration in the adult brain. (a) Following laser microlesion (yellow arrow in the +5 min panel) and subsequent

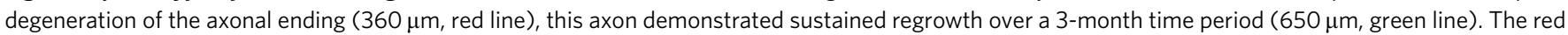
dotted line box indicates the area followed over time in the time-stamped panels. Insets at $+5 \mathrm{~d}$ and $+51 \mathrm{~d}$ show the growth cone. The dotted box at $+51 \mathrm{~d}$ refers to part of the axon shown in more detail in Fig. 5f. (b) Approximately 55\% of L6 axons (red) demonstrated regrowth over several weeks compared with only $\sim 20 \%$ of all other axons (blue; $P=0.01$, Pearson's $\chi^{2}$-test). (c) Injured axons that never reached the lesion site show only minimal regrowth. Bars show average maximum regrowth ( \pm s.e.m.) for a given time period as measured from the point of maximum retraction in the preceding days ( $n=29$ axons that did not reach the lesion site and $n=17$ that did reach the lesion site; ${ }^{\star \star} P<0.01$, Wilcoxon's rank sum test). (d) Regrowth in juvenile animals ( $n=7$ axons). Scale bar, $50 \mu \mathrm{m}$.

undergo dramatic regeneration (Fig. $4 \mathrm{~b}$ and Supplementary Movie 2 ), we find a general inability of other cortical axons to mount a regrowth response following axotomy (Fig. 4b). In addition to regeneration from the cut ends we also looked for evidence of collateral sprouting, the appearance of de novo branch formation along the primary axon shaft, which is common in the spinal cord after corticospinal ${ }^{29}$ and sensory neuron damage $e^{30,31}$. Collateral sprouting was not observed in our experiments, again indicating differences between axonal populations in different parts of the CNS in their response to injury. Furthermore, we have characterized the similarities and differences between regeneration in the adult and juvenile cerebral cortex. Severed axons in the juvenile brain had higher growth potential than those in adults; in addition, their mode of growth differed quite extensively and mimicked mechanisms seen in earlier developments, such as simultaneous growth and pruning ${ }^{23}$ (Fig. 4d).

Previous studies showed that severed retinal ganglion cells are able to grow on peripheral grafts and innervate both natural (superior colliculus) and non-natural targets (cerebellum) ${ }^{32}$. The synapses formed by regenerating axons were indistinguishable from those formed by non-lesioned axons ${ }^{32}$. We find that cortical axon regrowth in the grey matter is associated with ectopic bouton addition (Figs 4a, 5 and 6), suggesting the formation of connections with new targets. Remarkably, these new synaptic sites were added at comparable pre-injury densities (Fig. 5e), providing in vivo evidence for the existence of neuron-intrinsic homeostatic programmes, which regulate synaptic numbers on regenerating axons.

Glial/myelin-derived factors at the lesion site, together with intrinsic factors, are thought to be the main cause of the inability of CNS axons to regrow after injury. Deposition of CS in the cortex coincides with the end of critical periods of plasticity during development ${ }^{33}$, and enzymatic removal of CS results in increased sprouting and axonal growth in a range of injury paradigms $s^{5,34}$. In our paradigm, we show an increased propensity for regrowth in L6 axons, with the majority of other cortical axons unable to mount a regenerative response. Irrespective of the cortical axon subclass injured, or regenerative response 
a
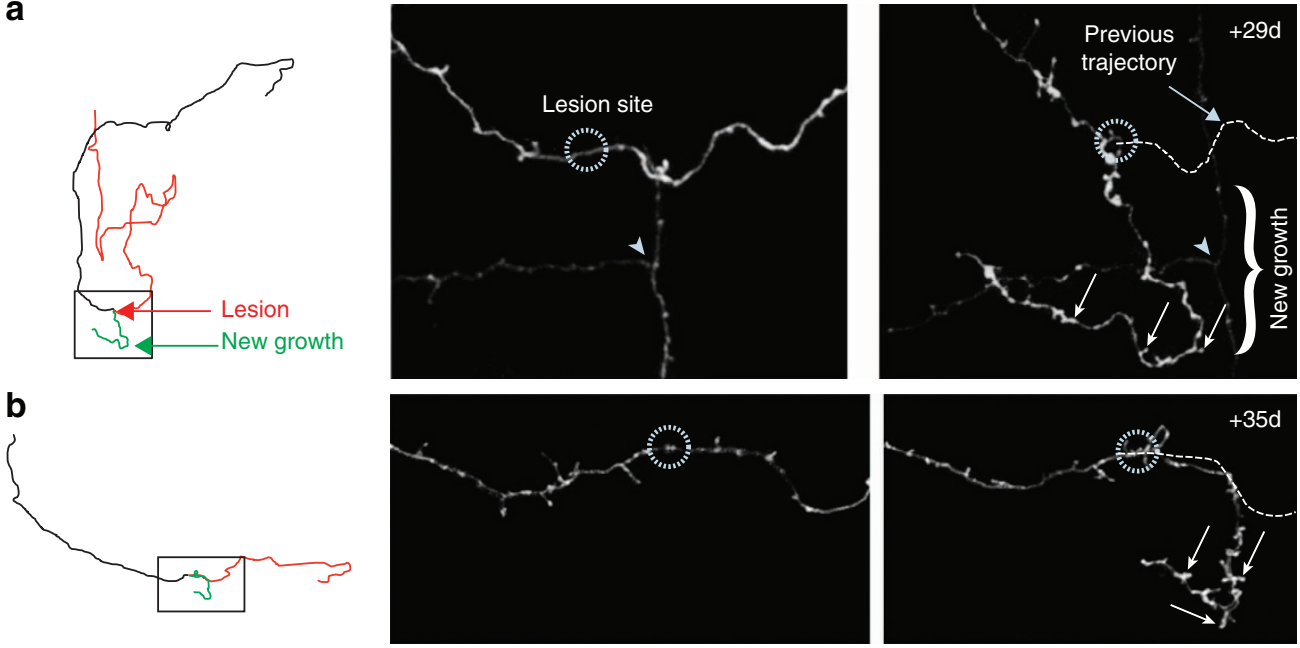

C

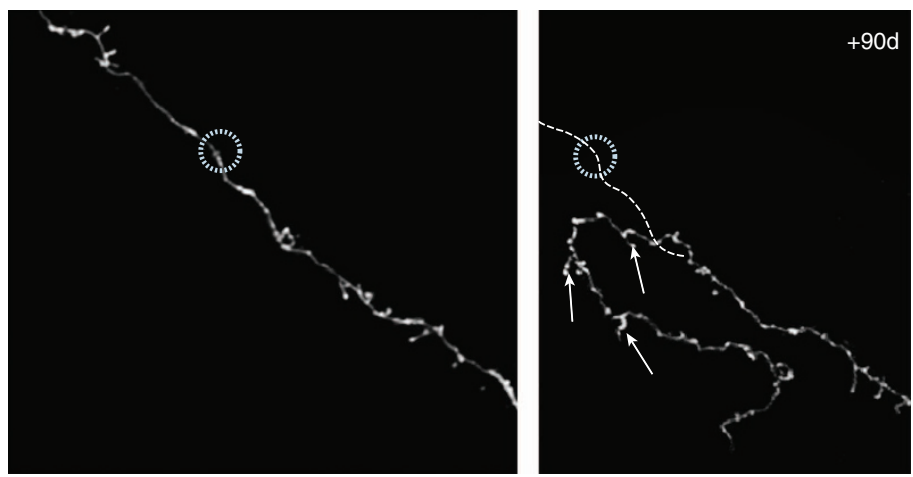

d

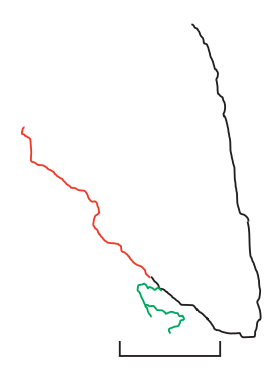

od
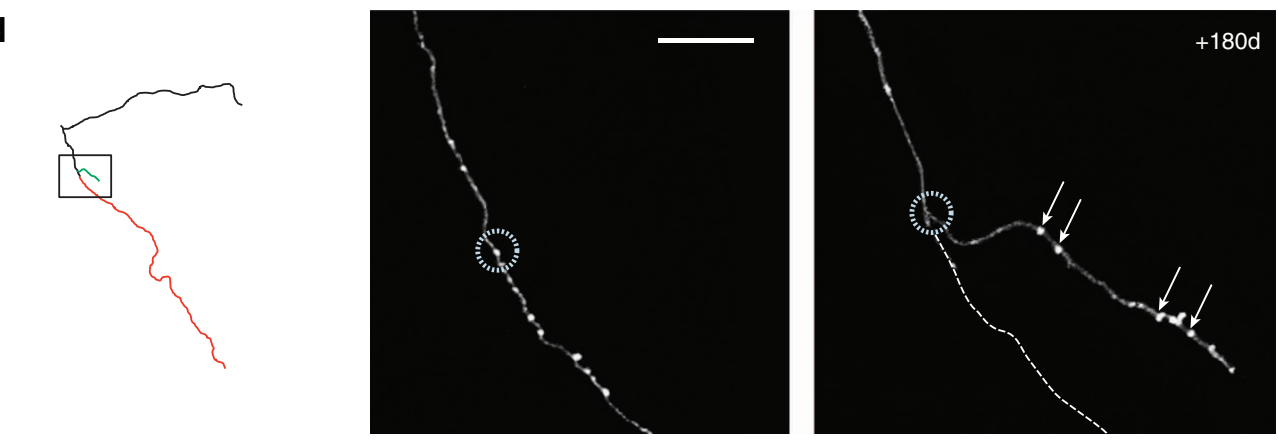

e

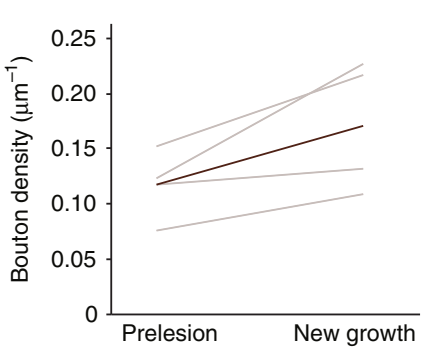

f
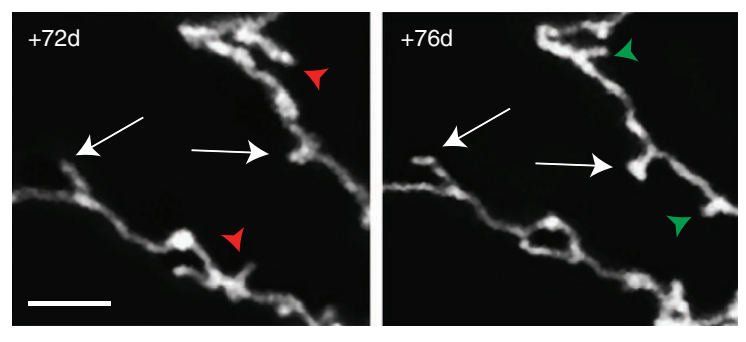

Figure 5 | Axon regrowth is associated with a reestablishment of normal bouton density contacting new targets. Regrowth of cortical axons typically avoided the lesion site and followed a different trajectory from the original axon (dotted line) at all times post lesion for both TB- (a-c) and en passant bouton- (d) rich axons; (a) +29 days, (b) +35 days, $(\mathbf{c})+90$ days and (d) +6 months post lesion. Reconstruction of the axon targeted for lesion is shown on the left in each case, with the distal portion in red and the regrown part in green. (e) Synaptic bouton analysis of L6 axons showing regrowth, demonstrates normal TB density in the regenerated portion compared with the pre-existing axonal shaft $(n=4$ axons, $P=0.13$, Wilcoxon's signed-rank test). Black line is the average. (f) Bouton formation (green arrowheads) and elimination (red arrowheads) on the regenerated portion of the axon in Fig. $4 \mathrm{a}$, same region as dotted boxed area at $+51 \mathrm{~d}$. White arrows indicate stable boutons, green and red arrowheads indicate bouton gains and losses, respectively. Scale bar, $10 \mu \mathrm{m}$ in $\mathbf{a}-\mathbf{d}$ and $5 \mu \mathrm{m}$ in $\mathbf{f}$. 

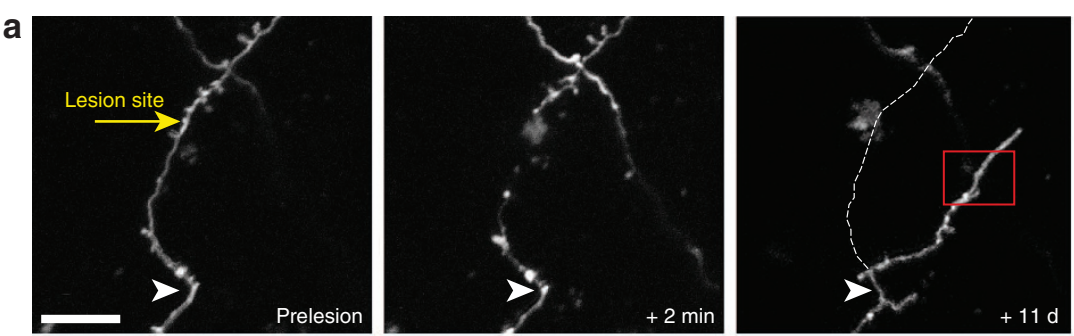

b
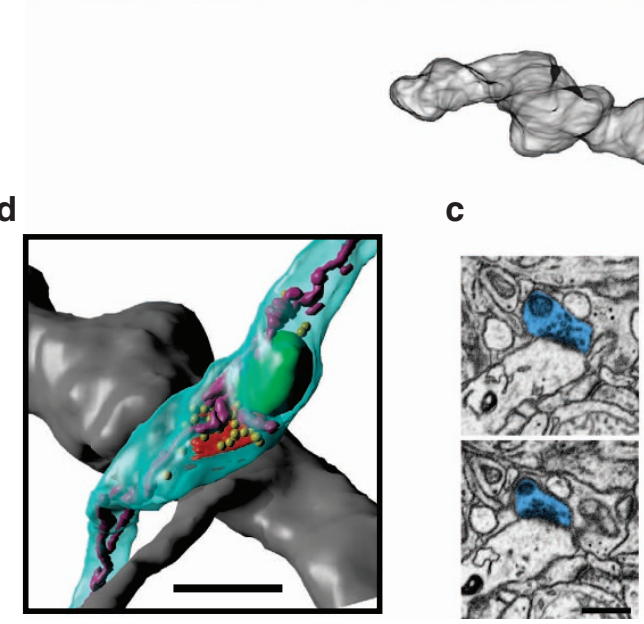

Figure 6 | Correlated 2P FIB-SEM reconstruction of a regrowing axon. (a) In vivo 2P imaging of axon regeneration after laser axotomy. The dotted line at $11 \mathrm{~d}$ shows the previous axon trajectory. (b) Three-dimensional rendering of the regenerated segment highlighted by the red box in a from FIB-SEM reconstruction. Note in the black box a newly formed synapse. (c) Two FIB-SEM images through the newly formed bouton (blue) that can be seen synapsing on the dendritic shaft. (d) High magnification of the black box in $\mathbf{b}$, showing the same bouton as in $\mathbf{c}$. In $\mathbf{b}$ and $\mathbf{d}$, the cytoplasm of the axon is represented in light blue, mitochondria in green, synaptic vesicles in yellow, synapse in red and endoplasmic reticulum in purple. The postsynaptic dendrite is shown in grey. Scale bars, $15 \mu \mathrm{m}$ in $\mathbf{a}, 1 \mu \mathrm{m}$ in $\mathbf{b}$ and $0.5 \mu \mathrm{m}$ in $\mathbf{c}$ and $\mathbf{d}$

observed, here we show a clear lack of sustained glial response at the lesion site or at the surviving axonal stump with a transient astrocytic and microglial response and no CS deposition (Fig. 2). Although it is still possible that if additional (known or unknown) inhibitory factors in the CNS were to be removed all neurons would regenerate, our results support the notion that rather than a local, glial-dependent inhibition of growth, an intrinsic inability of the neurons to reinitiate axonal growth is more likely ${ }^{4}$. This may be important when we consider the efficacy of therapeutic intervention following acquired brain injury ${ }^{35}$.

\section{Methods}

Animals. Adult male mice ( $n=42,12-14$ weeks) and early postnatal mice $(n=13$, PND 25-31) from Thy1-GFP-M ${ }^{13}$ and Thy1-GFP L15 ${ }^{14}$ (with cytosolic and membrane targeted GFP, respectively) were used for all imaging experiments unless otherwise specified. Mice were housed with littermates in standard individually ventilated caging and were maintained in a $12 \mathrm{~h}$ light-dark cycle with access to food and water ad libitum. All experiments involving live animals were conducted by researchers holding a UK personal license and were conducted in accordance with the Animals (Scientific Procedures) Act 1986 (UK) and associated guidelines.

Surgery. Cranial windows were surgically implanted overlying barrel cortex according to previously described methods ${ }^{36}$. Briefly, mice were anaesthetized with a ketamine-xylazine intraperitoneal (i.p.) injection $\left(0.083 \mathrm{mg} \mathrm{g}^{-1} \mathrm{ketamine}\right.$ $0.0078 \mathrm{mg} \mathrm{g}^{-1}$ xylazine). The animals were then administered intramuscular dexamethasone $\left(0.02 \mathrm{ml}\right.$ at $\left.4 \mathrm{mg} \mathrm{ml}^{-1}\right)$ to limit inflammation response, and subcutaneous bupivacaine $\left(1 \mathrm{mg} \mathrm{kg}^{-1}\right)$, a local anaesthetic. Once the skull was exposed, a few drops of lidocaine ( $1 \%$ solution) were applied on its surface. The glass coverslip that seals the window was placed directly over the dura and the bone edges, with a thin layer of agarose in between, and was sealed with dental cement. Mice were allowed to recover for 10-15 days before the start of the imaging protocol.
Imaging. A purpose built microscope equipped with a tunable Coherent Ti:Sapphire laser and PrarieView acquisition software was used for all in vivo imaging experiments. Mice were anaesthetized with ketamine-xylazine $(0.083$ and $0.0078 \mathrm{mg} \mathrm{g}^{-1}$, i.p.) and were secured to a fixed support under the microscope. The eyes were coated with Lacri-lube (Allergan) to prevent dehydration, an underlying heat pad used to maintain body temperature $\left(37^{\circ} \mathrm{C}\right)$, and rehydration administered with isotonic saline solution (i.p.) as required during long imaging sessions. Depth of anaesthesia was closely monitored. An Olympus $4 \times$ with a 0.13 numerical aperture objective was used to identify characteristic blood vessel patterns and to reliably relocate previously imaged areas of the cortical neuropil. An Olympus $40 \times, 0.80$ numerical aperture water immersion objective was used to acquire the images (typically $100 \times 100 \mu \mathrm{m}$ field of view, $512 \times 512$ pixels). A pulsed $910-\mathrm{nm}$ laser beam was used, never exceeding $70 \mathrm{~mW}$, on the back focal plane. Each imaging session typically lasted for 60-90 min, during which time up to 40 image stacks ( $1 \mu \mathrm{m}$ step size) were collected. Cortical axons were identified on the basis of morphology and those with clear axonal endings of at least $500 \mu \mathrm{m}$ in length were judged suitable for analysis. Where possible, two to three non-overlapping axons were selected for lesion in each preparation (Fig. 3). We routinely visualize the overlying blood vessels using dextran tracers to prevent damaging the surrounding microvasculature (Fig. 1b).

Laser-mediated microlesions in the intact mouse brain. A total of 107 axons were lesioned and followed chronically for at least two consecutive time points (83 axons in the adult brain, 48 for more than 2 weeks; and 24 axons in the developing brain, 12 for more than 2 weeks). The laser power, wavelength, duration and depth of penetration of laser firing were optimized to induce reproducible lesions. The imaging laser was manipulated to deliver a single spot scan using a binary mask of $4 \mu \mathrm{m}$ diameter, $\sim 600 \mathrm{~mW}$ at the back focal plane (that is, one order of magnitude higher than the power used for imaging), $800 \mathrm{~nm}$ and $30 \mu \mathrm{s}$ dwell time to the identified axon, which routinely resulted in severing the axon shaft. Lesions were conducted in the upper layers of the cortex, to a depth of $50 \mu \mathrm{m}$ beneath the pial surface. A dextran tracer ( $5 \mathrm{mg} \mathrm{ml}^{-1}$ solution; Texas Red, Molecular Probes) was injected into the tail vein, which labelled the vasculature to avoid targeting axonal lesions close to blood vessels. Lesions were made as far apart as possible, and always at least $300 \mu \mathrm{m}$ apart. 
Cortical axons were imaged at variable intervals before and after lesion for as long as window clarity was maintained. Mice were sacrificed at defined time points post lesion (1, 3, 6 and 12 months) for immunohistochemical studies or where the health of the mouse became compromised (maximum age post lesion 12 months). During one of the imaging sessions, overlapping low-magnification overviews of the labelled neuropil were collected to aid identification of axons in the fixed brain (Fig. 3).

At the conclusion of each experiment, mice were administered a lethal dose of ketamine-xylazine and transcardially perfused with $40 \mathrm{ml}$ of ice-cold $4 \%$ paraformaldehyde over $10 \mathrm{~min}$. The brain was dissected, post-fixed overnight at $4{ }^{\circ} \mathrm{C}$ in the same fixative, transferred to $0.01 \mathrm{M}$ PBS containing $0.02 \%$ azide and stored in the fridge. Where a significant interval of time had elapsed between the final live-imaging session and fixation, a final series of images were collected from the fixed whole mount brain to record any alterations in the labelled circuitry.

Immunocytochemistry. Brains were cryoprotected in 30\% sucrose for a minimum of $48 \mathrm{~h}$ at $4{ }^{\circ} \mathrm{C}$. Serial free-floating $40-\mu \mathrm{m}$ tangential sections (across the face of the window) were collected using a Leica cryostat. Sections were imaged at low magnification to allow visualization of surface blood vessels, which enabled digital alignment of the sections with in vivo images of blood vessels, the labelled neuropil and axons of interest (Adobe Photoshop Suite, Fig. 3). The identified sections were then processed for a range of antibodies using standard immunofluorescence protocols and were visualized on a Leica SP5 confocal microscope. Briefly, sections were blocked in $2 \%$ bovine serum albumin, $5 \%$ normal donkey serum in PBS containing $0.5 \%$ Triton X-100, followed by overnight incubation in the following primary antibodies; rabbit-anti-GFAP (DAKO; z0334, 1:2,000), goat-anti-Ibal (AbCam; ab5076, 1:500) and mouse-anti-CS proteoglycan (Sigma; clone 56, 1:500). Those sections labelled with Ibal required citrate buffer antigen retrieval $(0.2 \mathrm{M}$, $\mathrm{pH} 6.0,20 \mathrm{~min}$ at $95^{\circ} \mathrm{C}$ ) with subsequent boosting of quenched GFP with a chicken-anti-GFP antibody (Novus; NB100-1614, 1:5,000). The appropriate secondary antibodies were sourced from Jackson Immunoresearch and Molecular Probes.

Correlated 2P EM using focused ion beams. After the last in vivo imaging session, the animals were deeply anaesthetized as described above and transcardially perfused with $2 \%$ paraformaldehyde (wt $/ \mathrm{vol}$ ) and $2.5 \%$ glutaraldehyde ( $\mathrm{vol} / \mathrm{vol}$ ) in $0.1 \mathrm{M}$ phosphate buffer (pH 7.4). Vibratome sections of $60 \mu \mathrm{m}$ thickness were cut tangential to the surface of the cortex and parallel to the imaging plane of the $2 \mathrm{P}$ microscope. These were then imaged and a small $\left(30 \times 30 \mu \mathrm{m}^{2}\right)$ square was burned with the laser, around the region of interest. This fiducial mark was visible after resin embedding and was used to locate the imaged axon 37,38

The section was then stained with $1.5 \%$ potassium ferrocyanide and $1 \%(\mathrm{w} / \mathrm{v})$ osmium tetroxide in $0.1 \mathrm{M}$ cacodylate buffer $(0.1 \mathrm{M}, \mathrm{pH} 7.4)$, and then with $1 \%$ $(\mathrm{w} / \mathrm{v})$ uranyl acetate in double distilled water. It was then dehydrated with increasing concentrations of ethanol and embedded in Durcupan resin. This was then cured at $60^{\circ} \mathrm{C}$ for $48 \mathrm{~h}$. The region of interest was then cut from the rest of the section, and stuck to a blank resin block. This was further trimmed with glass knives in an ultramicrotome, placed on a metal stub, gold coated in a plasma vaporation system (Cressington) and placed inside a FIB-SEM (NVision 40 FIBSEM, Zeiss NTS). This was used to image the face of the block at exactly the position above the laser marks. A total of $\sim 3,000$ serial images were collected, with $12 \mathrm{~nm}$ distance between each image. The pixel size was $6 \mathrm{~nm}$. The final image series was analysed in the Fiji software package (http://fiji.sc/wiki/index.php/Fiji). Labelled elements of interest-axons, boutons, mitochondria, endoplasmic reticulum, synaptic vesicles and dendrites contacting labelled boutons-were manually segmented using the TrakEM2 programme (Fiji software package). The reconstructed model was rendered in the Blender software (version 2.57, Blender Foundation, http://www.blender.org).

Data analysis. In vivo 2P images were processed using Matlab (synaptic remodelling), Image J (length quantification, file conversions), Adobe Photoshop (section alignment, brightness and contrast, image rotation) or Neurolucida (axonal arbour tracing) as required. Figures were prepared using Microsoft Office Suite and Adobe Illustrator. Axons were classified according to morphological criteria as previously described ${ }^{8}$. Briefly, axons were grouped as L6 if Terminaux Bouton (TB) density was $>0.07$ per $\mu \mathrm{m}$. All other axons were grouped as L2/3/5 and TCA.

We defined attempted regrowth if the elongation from the cut stump reached the lesion site and also exceeded twice the maximum noise measurement (that is, $>6 \mu \mathrm{m}$; the maximum difference in measurements between fiducial points over repeated imaging sessions is $3 \mu \mathrm{m}^{8}$ ). For the density measurements in Fig. 5e, we used comparable lengths of the axon from the axonal tip, from both the prelesion ending and the length of axonal regrowth, whichever was shortest. For nonregrowing axons to be included in the calculations they had to be followed for more than two weeks without meeting the criteria above.

Terminaux boutons on L6 axons were manually traced and correlated between sessions using custom analysis software ${ }^{36}$. TBs $(1-5 \mu \mathrm{m})$ were annotated and scored as either stable, lost or gained according to stringent criteria modified from ref. 36. A comparison of $\mathrm{TB}$ annotations made in the same axons imaged in the living mouse immediately before and after fixation in the whole mount brain revealed no significant differences in synaptic density (Fig. 3f,g).

En passant bouton-rich axons were analysed using EPBscore ${ }^{39}$, a Matlab custom-built software.

All statistical analysis was performed either in the Matlab suite or Microsoft Excel. Unless stated otherwise, all measurements are given as mean \pm s.e.m. Wilcoxon's signed-rank test was used for all the comparisons unless otherwise stated. Results were considered significant when $P<0.05$. In the figures, ${ }^{\star} P<0.05$ and ${ }^{* *} P<0.01$.

\section{References}

1. Tuszynski, M. H. \& Steward, O. Concepts and methods for the study of axonal regeneration in the CNS. Neuron 74, 777-791 (2012).

2. Darian-Smith, C. Synaptic plasticity, neurogenesis, and functional recovery after spinal cord injury. Neuroscientist 15, 149-165 (2009).

3. Bradbury, E. J. \& McMahon, S. B. Spinal cord repair strategies: why do they work? Nat. Rev. Neurosci. 7, 644-653 (2006).

4. Bradke, F., Fawcett, J. W. \& Spira, M. E. Assembly of a new growth cone after axotomy: the precursor to axon regeneration. Nat. Rev. Neurosci. 13, 183-193 (2012).

5. Bradbury, E. J. et al. Chondroitinase $\mathrm{ABC}$ promotes functional recovery after spinal cord injury. Nature 416, 636-640 (2002).

6. Alto, L. T. et al. Chemotropic guidance facilitates axonal regeneration and synapse formation after spinal cord injury. Nat. Neurosci. 12, 1106-1113 (2009).

7. Pearse, D. D. et al. cAMP and Schwann cells promote axonal growth and functional recovery after spinal cord injury. Nat. Med. 10, 610-616 (2004).

8. De Paola, V. et al. Cell type-specific structural plasticity of axonal branches and boutons in the adult neocortex. Neuron 49, 861-875 (2006).

9. Kim, J. E., Liu, B. P., Park, J. H. \& Strittmatter, S. M. Nogo-66 receptor prevents raphespinal and rubrospinal axon regeneration and limits functional recovery from spinal cord injury. Neuron 44, 439-451 (2004).

10. Hawthorne, A. L. et al. The unusual response of serotonergic neurons after CNS Injury: lack of axonal dieback and enhanced sprouting within the inhibitory environment of the glial scar. J. Neurosci. 31, 5605-5616 (2011).

11. Blizzard, C. A. et al. Focal damage to the adult rat neocortex induces wound healing accompanied by axonal sprouting and dendritic structural plasticity. Cereb. Cortex. 21, 281-291 (2011).

12. Steward, O., Zheng, B. \& Tessier-Lavigne, M. False resurrections: distinguishing regenerated from spared axons in the injured central nervous system. J. Comp. Neurol. 459, 1-8 (2003).

13. Feng, G. et al. Imaging neuronal subsets in transgenic mice expressing multiple spectral variants of GFP. Neuron 28, 41-51 (2000).

14. De Paola, V., Arber, S. \& Caroni, P. AMPA receptors regulate dynamic equilibrium of presynaptic terminals in mature hippocampal networks. Nat. Neurosci. 6, 491-500 (2003).

15. Ylera, B. et al. Chronically CNS-injured adult sensory neurons gain regenerative competence upon a lesion of their peripheral axon. Curr. Biol. 19, 930-936 (2009).

16. Allegra Mascaro, A. L., Sacconi, L. \& Pavone, F. S. Multi-photon nanosurgery in live brain. Front Neuroenergetics 2, 21 (2010).

17. O'Brien, G. S. et al. Developmentally regulated impediments to skin reinnervation by injured peripheral sensory axon terminals. Cur. Biol. 19, 2086-2090 (2009).

18. Nimmerjahn, A., Kirchhoff, F. \& Helmchen, F. Resting microglial cells are highly dynamic surveillants of brain parenchyma in vivo. Science 308, 1314-1318 (2005).

19. Sun, D. \& Jakobs, T. C. Structural remodeling of astrocytes in the injured CNS Neuroscientist 18, 567-588 (2011).

20. Knott, G., Rosset, S. \& Cantoni, M. Focussed ion beam milling and scanning electron microscopy of brain tissue. J. Visual. Exper. 53, e2588 (2011).

21. Galtrey, C. M. \& Fawcett, J. W. The role of chondroitin sulfate proteoglycans in regeneration and plasticity in the central nervous system. Brain. Res. Rev. 54, 1-18 (2007).

22. Kwok, J. C. et al. Chondroitin sulfates in the developing rat hindbrain confine commissural projections of vestibular nuclear neurons. Neural Dev. 7, 6 (2012).

23. Portera-Cailliau, C., Weimer, R. M., De Paola, V., Caroni, P. \& Svoboda, K. Diverse modes of axon elaboration in the developing neocortex. PLoS Biol. 3, e272 (2005).

24. Bareyre, F. M. et al. In vivo imaging reveals a phase-specific role of STAT3 during central and peripheral nervous system axon regeneration. Proc. Natl Acad. Sci. USA 108, 6282-6287 (2011).

25. Yanik, M. F. et al. Neurosurgery: functional regeneration after laser axotomy. Nature 432, 822 (2004).

26. Farrar, M. J. et al. Chronic in vivo imaging in the mouse spinal cord using an implanted chamber. Nat. Methods 9, 297-302 (2012).

27. Allegra Mascaro, A. L. et al. In vivo single branch axotomy induces GAP43dependent sprouting and synaptic remodeling in cerebellar cortex. Proc. Natl Acad. Sci. USA (e-pub ahead of print 10 June 2013; doi:10.1073/pnas.1219256110). 
28. Canty, A. J. et al. Synaptic elimination and protection after minimal injury depend on cell type and their prelesion structural dynamics in the adult cerebral cortex. J. Neurosci. 33, 10374-10383 (2013).

29. Liu, K. et al. PTEN deletion enhances the regenerative ability of adult corticospinal neurons. Nat. Neurosci. 13, 1075-1081 (2010).

30. Kerschensteiner, M., Schwab, M. E., Lichtman, J. W. \& Misgeld, T. In vivo imaging of axonal degeneration and regeneration in the injured spinal cord. Nat. Med. 11, 572-577 (2005).

31. Dray, C., Rougon, G. \& Debarbieux, F. Quantitative analysis by in vivo imaging of the dynamics of vascular and axonal networks in injured mouse spinal cord. Proc. Natl Acad. Sci. USA 106, 9459-9464 (2009).

32. Aguayo, A. J. et al. Synaptic connections made by axons regenerating in the central nervous system of adult mammals. J. Exp. Biol. 153, 199-224 (1990).

33. Pizzorusso, T. et al. Reactivation of ocular dominance plasticity in the adult visual cortex. Science 298, 1248-1251 (2002).

34. Koprivica, V. et al. EGFR activation mediates inhibition of axon regeneration by myelin and chondroitin sulfate proteoglycans. Science 310, 106-110 (2005).

35. Zorner, B. \& Schwab, M. E. Anti-Nogo on the go: from animal models to a clinical trial. Ann. NY Acad. Sci. 1198(Suppl 1), E22-E34 (2010).

36. Holtmaat, A. et al. Long-term, high-resolution imaging in the mouse neocortex through a chronic cranial window. Nat. Protoc. 4, 1128-1144 (2009).

37. Bishop, D. et al. Near-infrared branding efficiently correlates light and electron microscopy. Nat. Methods 8, 568-570 (2011).

38. Maco, B. et al. Correlative in vivo 2 photon and focused ion beam scanning electron microscopy of cortical neurons. PLoS ONE 8, e57405 (2013).

39. Grillo, F. W. et al. Increased axonal bouton dynamics in the aging mouse cortex. Proc. Natl Acad. Sci. USA 110, E1514-E1523 (2013).

\section{Acknowledgements}

We thank Derron Bishop for sharing protocols and for help with the correlated 2P EM experiments; Jana Kalz and Hamlata Dechwland for help with experiments and analysis; Pico Caroni, Carlos Portera-Cailliau, James Fawcett and Martin Raff for comments on a previous version of the manuscript and Anthony Lewis for help with the figures. This work was supported by the Medical Research Council.

\section{Author contributions}

A.J.C. and V.D.P. designed the experiments; A.J.C. and V.D.P. performed surgeries, image acquisition, data analysis and wrote the manuscript. A.J.C., J.J. and L.H. performed the experiments with juveniles. L.H. performed the NIRB experiments for the correlated 2P/FIB-SEM reconstruction. J.J. and G.E.L. helped with the analysis. M.B. and G.K. performed the FIB-SEM reconstructions. All authors revised the manuscript.

\section{Additional information}

Supplementary Information accompanies this paper at http://www.nature.com/ naturecommunications

Competing financial interests: The authors declare no competing financial interests.

Reprints and permission information is available online at http://npg.nature.com/ reprintsandpermissions/

How to cite this article: Canty, A. J. et al. In-vivo single neuron axotomy triggers axon regeneration to restore synaptic density in specific cortical circuits. Nat. Commun. 4:2038 doi: 10.1038/ncomms3038 (2013).

(c) (1) $\ominus$ This work is licensed under a Creative Commons Attributioncc. this license, visit http://creativecommons.org/licenses/by-nc-nd/3.0/ 